\title{
Sociedades patogénicas y locura colectiva: una mirada crítica a la normalidad
}

\author{
Enric Garcia Torrents
}

Resumen: El presente artículo aborda la necesidad de replantear los servicios de atención a la salud mental desde una perspectiva social, subrayando el impacto de la desigualdad y otros determinantes en el sufrimiento de las personas, a la vez que plantea una crítica al rol del modelo biomédico actual en el control de la población y el mantenimiento de un sistema socio-económico a la vez desquiciado y desquiciante.

Palabras clave: psiquiatría social, psicología social, salud colectiva

\begin{abstract}
The present article addresses the need to rethink mental health care services from a social perspective, highlighting the impact of inequality and other determinants on people's suffering, while posing a critique of the role of the current biomedical model in controlling the population and maintaining a socio-economic system that is both unhinged and unhinging.
\end{abstract}

Key words: social psychiatry, social psychology, collective health

"Estamos en guardia contra las enfermedades contagiosas del cuerpo, pero somos exasperadamente descuidados cuando se trata de las aún más peligrosas enfermedades colectivas de la mente."

"We are on guard against contagious diseases of the body, but we are exasperatingly careless when it comes to the even more dangerous collective diseases of the mind."

C. G. Jung, Collected Works Vol. 18

Aunque los problemas de salud mental tienen satisfactoria y significativa, actuando no sólo en la indudablemente una dimensión biológica, su naturaleza prevención de la aparición de problemas de salud mental va más allá del organismo, involucrando dimensiones sino también contribuyendo a la recuperación de sociales, culturales y psicológicas. Para bien o para mal, aquellos quienes los padecen (3).

estos problemas son un efecto de como organizamos nuestros asuntos a nivel colectivo: las circunstancias en las que nacemos, crecemos, vivimos, trabajamos y envejecemos. A pesar de ello, la psiquiatría biomédica, de orientación tecnológica y basada en el tratamiento individual, tiende a perder de vista los determinantes sociales $(1,2)$.

Empecemos, no obstante, viendo cómo el enfoque actual centrado en el paciente individual (tratando de identificar las causas últimas del malestar mental a nivel genético, neuropatológico y demás), puede y debe dar pié a un enfoque de salud pública basado en la población. En lugar de considerar sólo a la persona que se enfrenta al médico, desde esta perspectiva el alcance A lo largo del presente artículo se introducen se amplía a la familia, la red social, el vecindario y la sucintamente, basándonos en el estado actual de la comunidad, y a la sociedad en general, convirtiéndose literatura científica al respecto, los factores económicos, estas entidades colectivas en las que el individuo sociales y ambientales sobre los que deberíamos estar coexiste en el paciente a atender. prestando mayor atención para que todas las personas tengan la oportunidad de disfrutar de una vida más sana, 
Por supuesto, esto conlleva ir más allá de la psiquiatría y la medicina propiamente dichas, abrazando un enfoque completamente transdiciplinar e involucrando otros muchos sectores que repercuten en la salud, como pueden ser la educación, el lugar de trabajo, los medios de comunicación, el gobierno y el sistema de justicia (salus populi suprema lex esto, al fin y al cabo).

Conlleva también, por supuesto, ir más allá de la mera mitigación de los factores de riesgo y la promoción de aquellos que suponen una protección contra la enfermedad de las personas, atacando las causas fundamentales de los problemas a través del compromiso y las intervenciones socio-políticas con efectos distales sobre el bienestar y la salud, llegando a plantear un enfoque y vía de acción, en la medida de lo posible, total, con una visión clara de la dirección en la que nuestras sociedades deberían tender a moverse (4).

Un primer paso en este sentido es reconocer que, de igual modo que se ha demostrado firmemente que la salud física varía a lo largo de un gradiente social, la salud mental se correlaciona fuertemente con la posición que uno ocupa en la sociedad, viéndose desproporcionalmente afectados los colectivos más vulnerables, desfavorecidos y minoritarios de la misma, expuestos a condiciones estresantes crónicas como pueden ser la inseguridad laboral, económica y de vivienda, la pobreza relativa, la marginación, el aislamiento social, la falta de estatus y la violencia, todos ellos obstáculos que vienen a sumarse al muy probable padecimiento de condiciones adversas durante la infancia y la presencia de barreras de acceso a la atención debidas a factores culturales, financieros y de orientación sexual, entre otros (5).

Existen pruebas abrumadoras de que las desigualdades materiales tienen poderosos efectos psicológicos, y que las sociedades menos igualitarias tienen un efecto negativo en las personas, desde la educación hasta la esperanza de vida pasando por la salud mental (6-11). Sin ir más lejos, en España la probabilidad de sufrir enfermedades mentales crónicas, así como el riesgo de cometer suicidio, es mucho mayor entre las personas migrantes, las personas con empleos precarios y aquellas con menor nivel educativo, llegando a doblarse cuando se comparan las personas en paro con las empleadas (12, 13). La situación, lamentablemente, no ha hecho más que empeorar debido a las sucesivas crisis económicas y los recortes presupuestarios en políticas sociales, apreciándose un incremento significativo, especialmente en el colectivo más joven, en la incidencia de todo tipo de trastornos mentales, desde los problemas de ansiedad hasta el abuso y la dependencia del alcohol y otras drogas, pasando por alteraciones de la conducta, estados depresivos, trastornos neuróticos y de personalidad, y la psicosis (14-16).
Aunque las diferencias de género en las tasas de problemas mentales es una área aún muy poco investigada, datos a nivel internacional apuntan que las mujeres son aproximadamente un $75 \%$ más propensas que los hombres a reportar haber sufrido recientemente una depresión, y alrededor de un $60 \%$ más propensas a reportar un trastorno de ansiedad (17). Dada la devaluación patriarcal del trabajo doméstico y los cuidados no remunerados, y teniendo en cuenta además que a las mujeres se les paga menos, les resulta más difícil avanzar en su carrera profesional, suelen tener que hacer malabarismos con múltiples roles y son bombardeadas a través de los medios con imágenes de una aparente perfección, resultaría sorprendente si todo ello no tuviera un costo emocional evidente.

Estudios recientes apuntan que, de igual manera, las personas no-heterosexuales padecen desproporcionadamente no solo de angustia psicológica y trastornos mentales, sino también de otros problemas de salud debidos al estrés crónico causado por los prejuicios aún prevalentes en nuestra sociedad $(18,19)$.

Múltiples fuentes de desigualdad se entrelazan y tienen un impacto acumulativo, afectando desproporcionadamente a los mismos colectivos y produciendo modos únicos de opresión y discriminación. Alcanzar mayores niveles de igualdad en todos los sentidos, así como de cooperación y reciprocidad, potenciando la autonomía relacional y la participación democrática de todas las personas en la vida colectiva con el fin de reducir el peso de la jerarquía social, aumentar la cohesión y la paridad de oportunidades debería hallarse, pues, en el centro de todo impulso para la creación de una sociedad más cuerda y sana.

Conveniente listar ahora algunos de los factores repetidamente identificados en la literatura científica como desencadenantes del desarrollo y la aparición de reacciones psicóticas, así como otras formas de sufrimiento psicológico. Estos son: el estrés prenatal, el abuso infantil, la exposición a un ambiente urbano, el estado migratorio de la persona, la pertenecía a una minoría étnica, la experiencia repetida de exclusión y derrota social y, en general, la creación de vínculos de apego temeroso con los demás y la disociación como forma de hacer frente al hecho de vivir en un entorno familiar y social adverso $(20,21)$.

Las alucinaciones y los delirios, más que síntomas de una supuesta predisposición genética o alteración biológica, son reacciones comprensibles a los eventos y circunstancias de la vida (22). Esta es la explicación más parsimoniosa para el patrón de hallazgos observado, pues es muy poco probable que los genes que contribuyen a cierto tipo de desarrollo neurológico aberrante codifiquen también para la migración, la condición de minoría étnica desfavorecida, la crianza en 
entornos con un tamaño y densidad de población elevados, la homosexualidad, los problemas socioeconómicos de origen y demás condicionantes reconocidos (21).

En resumidas cuentas, existen un sinfín de circunstancias que afectan negativamente al bienestar de las personas, impiden que se forme o minan gradualmente su capacidad de resiliencia y autoestima, y pueden llegar a derrumbarlas en momentos de especial vulnerabilidad 0 ante eventos percibidos como abrumadores. Es más, debemos reconocer que nadie es ajeno al sufrimiento $\mathrm{y}$, en un momento $\mathrm{u}$ otro, todos podemos llegar a caer. De hecho, más que una falsa y muy insidiosa dicotomía entre personas enfermas y sanas, lo que se observa -más allá de la cronificación debida a la estigmatización, la exclusión social, la medicalización de la miseria y los daños causados por los propios tratamientos- es un continuo dinámico en el cual cada persona puede ocupar distintas posiciones a lo largo de su vida (23-25).

El hecho de que cada persona tiene su punto de quiebre ha sido conocido $\mathrm{y}$ explotado desde tiempos inmemoriales (26). Nótese, eso si, que las nuevas formulaciones del modelo de diátesis-estrés no tratan tanto de cómo una supuesta predisposición o vulnerabilidad del individuo interactúa con el ambiente y con los sucesos vitales para desencadenar trastornos psicológicos, sino de una susceptibilidad diferencial en que ciertas personas se muestran especialmente sensibles tanto a las experiencias negativas como las positivas (27). Señalar también que la intensidad del estrés ambiental necesaria para alcanzar el punto en que la persona irremediablemente se descompone varía no solo de un individuo a otro, sino que también depende de variables como pueden ser: el nivel de optimismo y las expectativas positivas para el futuro, el hecho de practicar ejercicio $y$ el nivel de aptitud $y$ condicionamiento físico, la aplicación de técnicas que permiten un mejor manejo del estrés, como pueden ser la meditación y la relajación, el replanteamiento consciente de las percepciones negativas, la elección de un estilo de vida saludable evitando la privación del sueño y el consumo de sustancias toxicas, una nutrición adecuada y, quizás sobretodo, el hecho de gozar de una red de apoyo social suficientemente sólida (28-32).

No es ni justo ni suficiente, en cualquier caso, poner la carga y culpa enteramente encima de la víctima de abusos o circunstancias desfavorables y pretender que sean las mismas personas quienes han sufrido o sufren situaciones de angustia, conflicto y soledad, y se hallan incrustadas en jerarquías sociales opresivas, alienantes y violentas las que cambien su comportamiento y mentalidad para lograr así aliviar el impacto de las condiciones sociales negativas en las que viven, reduciendo la sobrecarga alostática que padecen (33-35). Como tampoco lo es centrar la práctica totalidad de la atención y los recursos en el estudio de los supuestos factores genéticos, de relación gen-ambiente mediados por cambios epigenéticos del genoma, y neurológicos que pueden conferir mayor vulnerabilidad -exacerbando los sentimientos de insuficiencia y ansiedad en las personas afectadas-, a la vez que se descuida negligentemente la investigación y las intervenciones a nivel biopsicosocial y colectivo que contribuirían a prevenir y aliviar el sufrimiento (36).

Lo primero, sea como sea, es no hacer daño (primum non nocere). Es inconcebible que a día de hoy aún se estén produciendo sistemáticamente intervenciones en el campo del sufrimiento psíquico coercitivas, violentas, deshumanizantes y (re)traumatizantes que contribuyen a reforzar la impotencia aprendida, privando de prácticamente toda esperanza de recuperación a las personas afectadas atribuyendo sus males a causas genéticas y procesos neurodegenerativos por determinar, todo ello a la vez que se las aísla de su entorno y comunidad y se empeora y cronifica su condición con intervenciones farmacológicas neurotoxicas que, aplicadas más allá de su posible función paliativa a corto plazo, contribuyen al deterioro y la discapacidad de las afectadas en colusión con velados intereses económicos y la preservación de un estatus quo que apenas tiene nada que ver con la salud de las personas.

El acceso a una atención segura, respetuosa y eficaz es un derecho humano; lamentablemente, la atención disponible para las personas a las que se les ha diagnosticado un trastorno mental a menudo no reúne ninguna de estas tres características (37).

Esto no es así por negligencia ni descuido, por supuesto, sino por el simple hecho que considerar y tratar las enfermedades mentales como un problema químicobiológico individual supone enormes beneficios para todas las partes con intereses creados en el sistema socio-económico actual.

En primer lugar, refuerza el impulso hacia la individualización y la destrucción de los vínculos sociales, debilitando la capacidad de resistencia y lucha de la población. El discurso psicológico de corte biomédico hace hincapié en que los individuos asuman la responsabilidad de las deficiencias e injusticias que experimentan; esta situación, totalmente intencional, sirve para ofuscar la realidad y conducir a las personas a cuestionar sus propias capacidades mentales en lugar de enfrentarse a las instituciones y poderes fácticos que las someten, aceptando el sufrimiento como una deficiencia personal. El sistema necesita la connivencia de los profesionales de la salud mental en esta farsa como una especie de atrezo: los servicios psiquiátricos y de psicología -sin negar las buenas intenciones de los practicantes implicados- enmascaran la insuficiencia de otros recursos sociales y de gobierno dificultando la posibilidad de enfoques más complejos y responsables 
en cuestiones socio-económicas; el uso de la atención mental permite a los estados aparentar que se preocupan y ayudan a la población a superar sus problemas mientras en realidad suscitan su conformidad hacia las condiciones que los generan (38).

En segundo lugar, todo ello provee un mercado enormemente lucrativo en el cual las compañías farmacéuticas multinacionales pueden vender sus productos a una proporción cada vez mayor de la población (39-41).

En una sociedad hiper-estresada, extremadamente competitiva y materialista como la nuestra las llamadas enfermedades mentales no son meras aberraciones sino el resultado natural de condiciones sociales obscenas y un modo de vida que no está en consonancia con las necesidades humanas más básicas y genuinas. La normalidad en este contexto no es más que una “patología de la conformidad”, impulsada para pacificar a la población y sostener un sistema rapaz que vive de la opresión social y económica, la alienación, la mistificación de los individuos y la explotación sin freno del medio natural (42).

Estar plenamente adaptado a un contexto profundamente enfermo, forzado a encajar en un entorno alienante como si este fuera un verdadero lecho de Procusto, sin luchar, sufrir o presentar síntomas, desarrollando comportamientos desviados de la norma, no puede considerarse un hecho exento de problemática $(26,43-$ 46).

Estos dictámenes críticos con la sociedad se acercan al diagnóstico, especialmente popular entre los Un primer paso, cabe añadir, que tan solo requiere un movimientos contra-culturales, al considerar los poco de valentía y humildad.

\section{Referencias:}

1. Bracken, Pat, et al. "Psychiatry beyond the current paradigm." The British journal of psychiatry 201.6 (2012): 430-434.

2. Compton, Michael T., and Ruth S. Shim. "The social determinants of mental health." Focus 13.4 (2015): 419425.

3. Morgan, Craig Ed, Kwame Ed McKenzie, and Paul Ed Fearon. Society and psychosis. Cambridge University Press, 2008.

4. Wilkinson, Richard, and Kate Pickett. The inner level: how more equal societies reduce stress, restore sanity and improve everyone's well-being. Penguin Books, 2020.

5. Fisher, Matthew, and Fran Baum. "The social determinants of mental health: implications for research and health promotion." Australian \& New Zealand Journal of Psychiatry 44.12 (2010): 1057-1063.

6. Babones, Salvatore J. "Income inequality and population health: correlation and causality." Social science \& medicine 66.7 (2008): 1614-1626.

7. Burns, Jonathan K., Andrew Tomita, and Amy S. Kapadia. "Income inequality and schizophrenia: increased schizophrenia incidence in countries with high levels of income inequality." International Journal of Social Psychiatry 60.2 (2014): 185-196.

8. Marmot, Michael G. "Status syndrome: a challenge to medicine." Jama 295.11 (2006): 1304-1307.

9. Pickett, Kate E., and Richard G. Wilkinson. "Inequality: an underacknowledged source of mental illness and distress." The British Journal of Psychiatry 197.6 (2010): 426-428.

10. Ribeiro, Wagner Silva, et al. "Income inequality and mental illness-related morbidity and resilience: a systematic review and meta-analysis." The Lancet Psychiatry 4.7 (2017): 554-562. 
11. Singer, Burton H., Carol D. Ryff, and National Research Council. "The influence of inequality on health outcomes." New horizons in health: An integrative approach. National Academies Press (US), 2001.

12. Espino Granado, Antonio. "Crisis económica, políticas, desempleo y salud (mental)." Revista de la asociación española de neuropsiquiatría 34.122 (2014): 385-404.

13. Moreno-Küstner, B., and AI Masedo Gutierrez. "Economic crisis and mental health-findings from Spain." Die Psychiatrie 14.02 (2017): 95-102.

14. Frasquilho, Diana, et al. "Mental health outcomes in times of economic recession: a systematic literature review." BMC public health 16.1 (2015): 1-40.

15. Gili, Margalida, Javier García Campayo, and Miquel Roca. "Crisis económica y salud mental. Informe SESPAS 2014." Gaceta Sanitaria 28 (2014): 104-108.

16. Read, John. "Can poverty drive you mad?'Schizophrenia', socio-economic status and the case for primary prevention." New Zealand Journal of Psychology 39.2 (2010): 7-19.

17. Freeman, Daniel, and Jason Freeman. The stressed sex: Uncovering the truth about men, women, and mental health. Oxford University Press, 2013.

18. Flentje, Annesa, et al. "The relationship between minority stress and biological outcomes: A systematic review." Journal of Behavioral Medicine (2019): 1-22.

19. King, Michael, et al. "A systematic review of mental disorder, suicide, and deliberate self harm in lesbian, gay and bisexual people." BMC psychiatry 8.1 (2008): 70.

20. Lim, Caroline, Siow-Ann Chong, and Richard SE Keefe. "Psychosocial factors in the neurobiology of schizophrenia: a selective review." Ann Acad Med Singapore 38.5 (2009): 402-406.

21. Selten, Jean Paul, Jim van Os, and Elizabeth Cantor Graae. "The social defeat hypothesis of schizophrenia: issues of measurement and reverse causality." World Psychiatry 15.3 (2016): 294.

22. Read, John, and Nick Haslam. "Bad things happen and can drive you crazy." Models of madness: Psychological, social and biological approaches to schizophrenia (2004): 133.

23. DeRosse, Pamela, and Katherine H. Karlsgodt. "Examining the psychosis continuum." Current behavioral neuroscience reports 2.2 (2015): 80-89.

24. Van Os, Jim, et al. "A systematic review and meta-analysis of the psychosis continuum: evidence for a psychosis proneness-persistence-impairment model of psychotic disorder." Psychological medicine 39.2 (2009): 179.

25. Verdoux, Hélène, and Jim van Os. "Psychotic symptoms in non-clinical populations and the continuum of psychosis." Schizophrenia research 54.1-2 (2002): 59-65.

26. Huxley, Aldous. "Brave new world revisited" 1958.

27. Belsky, Jay, and Michael Pluess. "Beyond diathesis stress: differential susceptibility to environmental influences." Psychological bulletin 135.6 (2009): 885.

28. Carver, Charles S., Michael F. Scheier, and Suzanne C. Segerstrom. "Optimism." Clinical psychology review 30.7 (2010): 879-889.

29. Maté, Gabor. When the body says no: The cost of hidden stress. Vintage Canada, 2011.

30. Peterson, Christopher. "The future of optimism." American psychologist 55.1 (2000): 44.

31. Ratey, John J. Spark: The revolutionary new science of exercise and the brain. Little, Brown Spark, 2008.

32. Southwick, STEVEN M., et al. "Adaptation to stress and psychobiological mechanisms of resilience." Biobehavioral resilience to stress (2008): 91-116.

33. McEwen, Bruce S., and John C. Wingfield. "The concept of allostasis in biology and biomedicine." Hormones and behavior 43.1 (2003): 2-15.

34. Rapley, Mark, Joanna Moncrieff, and Jacqui Dillon, eds. De-medicalizing misery: Psychiatry, psychology and the human condition. Springer, 2011.

35. Speed, Ewen, Joanna Moncrieff, and Mark Rapley, eds. De-medicalizing misery II: society, politics and the mental health industry. Springer, 2014.

36. Moncrieff, Joanna. "Psychiatric drug promotion and the politics of neoliberalism." The British Journal of Psychiatry 188.4 (2006): 301-302.

37. Higgs, Rory Neirin "Reconceptualizing Psychosis: The Hearing Voices Movement and Social Approaches to Health" Health and Human Rights Journal, Volume 22 Number 1 (2020): 133-144.

38. Ratner, Carl. Psychology's Contribution to Socio-Cultural, Political, and Individual Emancipation. Springer Nature, 2019.

39. Moncrieff, Joanna. "Neoliberalism and biopsychiatry: A marriage of convenience." Liberatory psychiatry: Philosophy, politics and mental health 9 (2008): 235-256.

40. Moncrieff, Joanna. "Psychiatric diagnosis as a political device." Social Theory \& Health 8.4 (2010): 370-382.

41. Fisher, Mark. Capitalist realism: Is there no alternative?. John Hunt Publishing, 2009.

42. Maté, Gabor, The Myth of Normal: Illness and Health in an Insane Culture. Penguin Random House, 2021.

43. Fromm, Erich. The pathology of normalcy. Lantern Books, 2011. 
44. Fromm, Erich, and Leonard A. Anderson. The sane society. Routledge, 2017.

45. Morrall, Peter. Madness: Ideas about Insanity. Taylor \& Francis, 2017.

46. Ratner, C. "Pathological normalcy: The psychological dimension of alienation and a guiding construct for overcoming alienation." The Humanistic Psychologist 42.2 (2014).

47. Frances, Allen. "Saving normal: An insider's revolt against out-of-control psychiatric diagnosis, DSM-5, big pharma and the medicalization of ordinary life." Psychotherapy in Australia 19.3 (2013): 14.

48. Staub, Michael E. Madness is civilization: When the diagnosis was social, 1948-1980. University of Chicago Press, 2011.

49. Rose, Nikolas. Our psychiatric future. John Wiley \& Sons, 2018. 